\title{
Role of Transesophageal Echocardiography in the Prediction of Thromboembolism in Patients With Chronic Nonvalvular Atrial Fibrillation
}

\author{
Sadae Miyazaki, MD; Takahide Ito, MD; Michihiro Suwa, MD; \\ Tomomi Nakamura, MD; Ayaka Kobashi, MD; Yasushi Kitaura, MD
}

\begin{abstract}
The purpose of this study was to determine whether parameters derived from transesophageal echocardiography (TEE) could predict thromboembolism in patients with chronic nonvalvular atrial fibrillation (AF). Eighty-nine patients, mean age 66 \pm 9 years, who underwent TEE in 1996 to 1999 were studied. The clinical endpoint was a thromboembolic event, including transient ischemic attack (TIA). Sixty-seven patients (75\%) were anticoagulated with warfarin after TEE. After a follow-up period of $29 \pm 10$ months, 1 patient died suddenly, 4 had a thromboembolism, and 3 had a TIA; the annual embolic event rate was 3.3\%. Left atrial appendage (LAA) thrombus ( $86 \%$ vs $17 \%, \mathrm{p}<0.001$ ), LAA dysfunction (LAA velocity $<20 \mathrm{~cm} / \mathrm{s} ; 71 \%$ vs $25 \%, \mathrm{p}=0.009$ ), and severe LA spontaneous echo contrast ( $29 \%$ vs $2 \%, \mathrm{p}=0.002$ ) were more prevalent in patients with an embolic event than in those without. In patients with LAA thrombus, the annual event rate was $11 \%$ as compared with $1.2 \%$ in those without ( $\mathrm{p}=0.004$ ). On the Cox proportional hazards model analysis, LAA thrombus (chi-square 7.0, $\mathrm{p}=0.008$ ), severe LA spontaneous echo contrast (chi-square 7.0, $\mathrm{p}=0.008$ ), and LAA dysfunction (chi-square 5.9, $\mathrm{p}=0.015$ ) were significantly related to thromboembolism. Multivariate analysis revealed that LAA thrombus (chi-square 5.5, $\mathrm{p}=0.019$ ) and LAA dysfunction (chi-square $4.0, \mathrm{p}=0.045$ ) were the independent predictors. In conclusion, TEE parameters, particularly the presence of LAA thrombus, can be used to assess thromboembolic potential in patients with chronic nonvalvular AF. (Jpn Circ J 2001; 65: 874-878)
\end{abstract}

Key Words: Nonrheumatic atrial fibrillation; Thromboembolism; Transesophageal echocardiography

$\mathbf{N}$ onvalvular atrial fibrillation (AF) is considerably associated with the risk of thromboembolism and several clinical and echocardiographic parameters are involved, including age, ${ }^{1-3}$ prior thromboembolism, $1,3,4$ left atrial (LA) size $e^{1,5}$ and left ventricular function!,5 However, there are few reports in which variables derived from transesophageal echocardiography (TEE) are used to assess the risk of future thromboembolism.-10 Because of its proximity to the cardiac chambers, TEE can scope over the entire LA cavity, including the LA appendage (LAA), to assess the presence or absence of LA thrombus and can also measure LAA velocity, an analogue to LAA function. Therefore, we prospectively examined the prognostic value of TEE-derived parameters for thromboembolism in patients with chronic nonvalvular AF.

\section{Methods}

\section{Study Patients}

Eighty-nine patients with chronic nonvalvular AF who had undergone TEE between September 1996 and October 1999 were enrolled (60 men, 29 women, aged $66 \pm 9$ (mean \pm $\mathrm{SD})$ years). The diagnosis of chronic nonvalvular AF was

(Received April 17, 2001; revised manuscript received June 26, 2001; accepted July 10, 2001)

The Third Division, Department of Internal Medicine, Osaka Medical College, Takatsuki, Osaka, Japan

Mailing address: Takahide Ito, MD, The Third Division, Department of Internal Medicine, Osaka Medical College, 2-7 Daigaku-cho, Takatsuki City, Osaka 569-8686, Japan. E-mail: in3016@ poh.osakamed.ac.jp made from electrocardiographic and echocardiographic findings; AF was documented on 2 separate occasions (at least 1 month apart) by electrocardiography and there was no evidence of rheumatic changes in the mitral valve apparatus. Degenerative aortic valve and mitral annular calcification were not exclusion criteria in this study. The underlying cardiovascular disease was hypertensive heart disease in 37 patients (with a history of hypertension irrespective of echocardiographic evidence of cardiac hypertrophy), dilated cardiomyopathy in 13 , hypertrophic cardiomyopathy in 7 , coronary artery disease in 4 , mitral valve prolapse in 3 , constrictive pericarditis in 1 , and alcoholic heart disease in 1 ; the remaining 23 patients had no cardiovascular abnormality predisposing to AF ('lone' AF). Twenty-one patients $(15 \%)$ had a history of suspected cardiogenic thromboembolism.

\section{Echocardiography}

All patients gave informed consent to participate in the study before echocardiography was carried out. Transthoracic echocardiography was performed immediately before TEE; the LA dimension and left ventricular end-diastolic/ systolic dimensions were determined by the standard Mmode criteria ${ }^{11}$ and the left ventricular fractional shortening was calculated.

TEE was performed with a 5-MHz multiplane transducer attached to a commercially available echocardiographic system (SONOS 2500, Hewlett-Packard, Wilmington, DE, USA). Particular attention was paid to imaging the LA cavity and LAA to assess the presence or absence of spontaneous echo contrast and thrombus. LA spontaneous echo 
Table 1 Comparison of the Clinical and Echocardiographic Data Between Patients With and Without an Embolic Event During Follow-up

\begin{tabular}{|c|c|c|c|}
\hline & $\begin{array}{l}\text { Event present } \\
(n=7)\end{array}$ & $\begin{array}{c}\text { Event absent } \\
\quad(n=81)\end{array}$ & $p$ value \\
\hline Age (years) & $66 \pm 8$ & $66 \pm 9$ & 0.984 \\
\hline$\geq 75$ years & $1(14 \%)$ & $12(15 \%)$ & 0.970 \\
\hline Female & $1(14 \%)$ & $28(35 \%)$ & 0.273 \\
\hline History of hypertension & $3(43 \%)$ & $23(28 \%)$ & 0.421 \\
\hline History of stroke prior to TEE & $2(29 \%)$ & $19(23 \%)$ & 0.761 \\
\hline Anticoagulation during follow-up & $5(71 \%)$ & $61(75 \%)$ & 0.820 \\
\hline LA dimension $(\mathrm{cm})$ & $4.9 \pm 0.7$ & $4.9 \pm 0.7$ & 0.986 \\
\hline$\geq 5.0 \mathrm{~cm}$ & $3(43 \%)$ & $44(54 \%)$ & 0.560 \\
\hline LV end-diastolic dimension $(\mathrm{cm})$ & $5.2 \pm 1.0$ & $5.2 \pm 0.7$ & 0.849 \\
\hline LV fractional shortening (\%) & $34 \pm 14$ & $31 \pm 8$ & 0.370 \\
\hline$<25 \%$ & $1(14 \%)$ & $15(19 \%)$ & 0.781 \\
\hline Moderate to severe $M R$ & $0(0 \%)$ & $8(10 \%)$ & 0.383 \\
\hline$L A A$ velocity $(\mathrm{cm} / \mathrm{s})$ & $22 \pm 12$ & $32 \pm 20$ & 0.221 \\
\hline$<20 \mathrm{~cm} / \mathrm{s}$ & $5(71 \%)$ & $20(25 \%)$ & 0.009 \\
\hline LAA size $\left(\mathrm{cm}^{2}\right)$ & $5.8 \pm 2.2$ & $4.5 \pm 1.5$ & 0.034 \\
\hline$\geq 5.5 \mathrm{~cm}^{2}$ & $3(43 \%)$ & $18(22 \%)$ & 0.219 \\
\hline \multicolumn{4}{|l|}{ LA SEC } \\
\hline None & $2(29 \%)$ & $39(48 \%)$ & 0.319 \\
\hline Mild & $3(43 \%)$ & $40(49 \%)$ & 0.740 \\
\hline Severe & $2(29 \%)$ & $2(2 \%)$ & 0.002 \\
\hline LAA thrombus present & $6(86 \%)$ & $14(17 \%)$ & $<0.001$ \\
\hline
\end{tabular}

Values expressed as mean $\pm S D$. TEE, transesophageal echocardiography; LA, left atrial; LV, left ventricular; LAA, left atrial appendage; SEC, spontaneous echo contrast.

contrast was diagnosed if there were dynamic smoke-like echoes within the LA cavity, with a characteristic swirling motion distinct from white noise artifact, and was qualitatively graded as none, mild (when it was observed only in part of the LA cavity with a high gain control), and severe (when it was visible throughout the entire LA cavity even with a low gain control) $!^{12-14}$ Particular care was paid to differentiate LA/LAA thrombus from normal cardiac structures, such as the pectinate muscles.

LAA size and function were assessed by 2-dimensional and Doppler echocardiography. In the longitudinal plane, the maximal LAA area (the largest area during one cardiac cycle) was measured by computer-assisted planimetry from the tip of the wall between the left upper lobe pulmonary vein and the LAA to its junction with the main LA cavity, as described previously 15,16 The LAA emptying velocity was measured with a sample volume placed $1-2 \mathrm{~cm}$ into the mouth of the LAA, and averaged over 5 consecutive cycles. Because the LAA filling velocities were approximately equal in magnitude to the emptying velocities, they were not separately measured in this study.

\section{Follow-up}

The clinical endpoints included thromboembolic events, including stroke and embolism to viscera or an extremity. Transient ischemic attack (TIA) was also considered an embolic event. These endpoints were determined from hospital records and information from the primary physicians, or through interviewing the patient's family by phone. The follow-up period was defined as beginning on the day after TEE and ending on March 31, 2000.

Because of ethical considerations, we recommended anticoagulation therapy with warfarin to the patients, especially those with an apparent high risk of future embolism (ie, presence of LA thrombus and/or severe spontaneous echo contrast). The target range for the international normalized ratio (INR) was 1.8-2.5, and the primary physicians monitored the ratio. The patients who could not undergo anticoagulation for some reason were recommended to receive aspirin ( $81 \mathrm{mg} /$ day).

\section{Statistical Analysis}

The continuous and discrete variables between 2 groups were compared using the unpaired test and chi-square test, respectively. Cox proportional hazards model analysis (both univariate and multivariate) was performed to determine which clinical and echocardiographic variables were significantly related to embolic events. In this model, chisquare analysis was utilized to assess the relative predictive power. Cutoff points for age ( $\geq 75$ years vs $<75$ years), LA dimension $(\geq 5.0 \mathrm{~cm}$ vs $<5.0 \mathrm{~cm})$, left ventricular systolic shortening ( $<25 \%$ vs $\geq 25 \%)$, and LAA velocity $(<20 \mathrm{~cm} / \mathrm{s}$ vs $\geq 20 \mathrm{~cm} / \mathrm{s}$ ) were made according to the Stroke Prevention in Atrial Fibrillation (SPAF) III trial ${ }^{7}$ and others?,17 The cutoff point for LAA size $\left(\geq 5.5 \mathrm{~cm}^{2}\right.$ vs $\left.<5.5 \mathrm{~cm}^{2}\right)$ was determined as the 75 th percentile. The event-free curves were assessed by the Kaplan-Meier method, and the prognosis between 2 groups was compared using the log rank test. All data were expressed as the mean $\pm \mathrm{SD}$, and a $\mathrm{p}$ value $<0.05$ was considered significant.

\section{Results}

No patient was lost to follow-up. Sixty-seven patients $(75 \%)$ were receiving anticoagulation therapy after TEE. During the follow-up period of 29 months, 1 patient died suddenly, 3 had a stroke, 1 had emboli to viscera, and 3 had a TIA, which resulted in an annual rate of thromboembolism of $3.3 \%$. Three of 7 patients with thromboembolism died soon after the event. The patient who died suddenly, being diagnosed as hypertrophic cardiomyopathy, was excluded from the subsequent analyses. The predominant cardiovascular disease in the 7 patients with an embolic event was hypertensive heart disease in 3, coronary artery disease in 2 , hypertrophic cardiomyopathy in 1 , and lone $\mathrm{AF}$ in 1. 
Table 2 Determinants of Adverse Outcome With Cox Proportional Hazard Model: Univariate and Multivariate Analyses

\begin{tabular}{lccccc}
\hline \hline & \multicolumn{2}{c}{ Univariate } & & \multicolumn{2}{c}{ Multivariate } \\
\cline { 2 - 3 } \cline { 5 - 6 } & Chi-square & $p$ value & & Chi-square & $p$ value \\
\hline History of hypertension & 0.0 & 0.855 & & 0.3 & 0.605 \\
History of stroke prior to TEE & 0.0 & 0.825 & & 1.1 & 0.303 \\
Anticoagulation during follow-up & 0.0 & 0.961 & & 0.0 & 0.938 \\
LA dimension $\geq 5.0 \mathrm{~cm}$ & 0.3 & 0.594 & & 2.6 & 0.106 \\
LAA velocity $<20 \mathrm{~cm} / \mathrm{s}$ & 5.9 & 0.015 & & 4.0 & 0.045 \\
LAA size $\geq 5.5 \mathrm{~cm}^{2}$ & 1.3 & 0.264 & & 0.0 & 0.878 \\
Severe SEC & 7.0 & 0.008 & & 1.7 & 0.197 \\
LAA thrombus present & 7.0 & 0.008 & & 5.5 & 0.019 \\
\hline
\end{tabular}

Abbreviations as in Table 1.
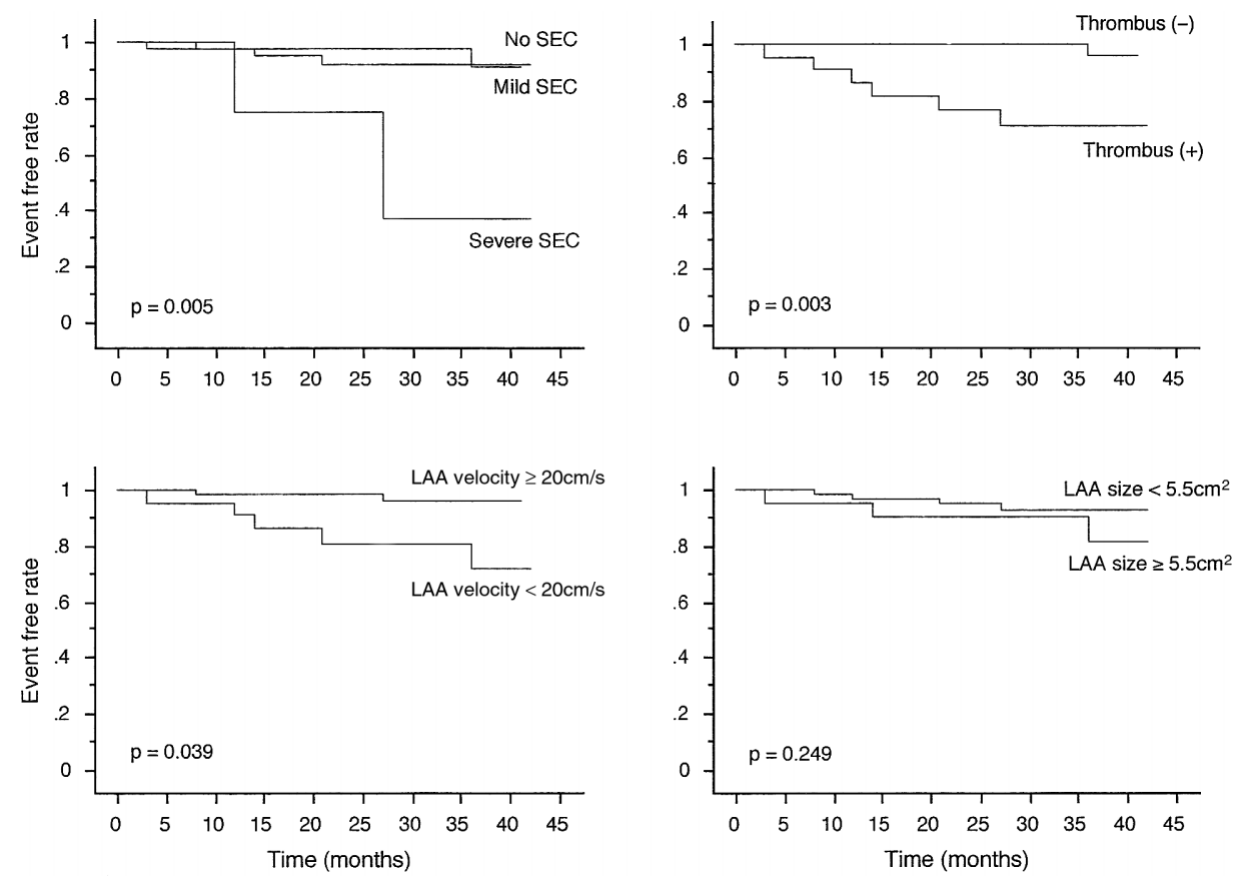

Fig 1. Kaplan-Meier curves in the patients divided into 2 or 3 groups based on various TEE-derived parameters. LAA, left atrial appendage; SEC, spontaneous echo contrast.

Table 1 is a comparison of the clinical and echocardiographic data between patients with and without an embolic event. There was no significant difference between the groups with regard to age, gender distribution or the proportion of patients who had a prior stroke or hypertension. Both groups received equivalent anticoagulant therapy during the follow-up. Five of 67 patients (7.5\%) undergoing anticoagulation therapy had a thromboembolic event, as compared with 2 of 21 patients $(9.5 \%)$ without this therapy. The LA dimension, left ventricular end-diastolic dimension, and left ventricular fractional shortening were comparable between the groups.

With regard to the parameters derived from TEE, the LAA size was larger and the LAA velocity was lower in patients with a clinical event than in those without. The patients with a LAA velocity $<20 \mathrm{~cm} / \mathrm{s}$ were more prevalent in the event-present group, but the proportion of patients with a LAA $\geq 5.5 \mathrm{~cm}^{2}$ in size was comparable between the groups. The LA spontaneous echo contrast was more severe and the incidence of LAA thrombus (all thrombi located in the LAA) was much higher in patients with an embolic event.
In patients with LAA thrombus, the annual event rate was $11 \%$ compared with $1.2 \%$ in those without $(\mathrm{p}=0.004)$. Among the 20 patients with LAA thrombus, 16 had received anticoagulant therapy after TEE and of these 16, 5 developed thromboembolism, resulting in an annual event rate of $13 \%$. In contrast, none of the patients without LAA thrombus had an embolic event during anticoagulation therapy. In patients with LAA dysfunction (LAA velocity $<20 \mathrm{~cm} / \mathrm{s}$ ), the annual event rate was $8 \%$, compared with $1.3 \%$ in those without $(\mathrm{p}=0.018)$. With the presence of spontaneous echo contrast, the event rate was $4.4 \%$ compared with $2 \%$ for those without $(\mathrm{p}=0.357)$.

Table 2 shows the results of Cox proportional hazards model analysis for the risk of thromboembolism. Because significant mitral regurgitation, increased age ( $\geq 75$ years) and female gender were noted in none or only 1 patient in the group with an embolic event and thus were unlikely to influence the outcome significantly, these factors were not incorporated in the model. In the univariate analysis, LAA thrombus, severe spontaneous echo contrast, and LAA velocity $<20 \mathrm{~cm} / \mathrm{s}$ were significantly related to the outcome, and multivariate analysis revealed that LAA thrombus and 
LAA velocity $<20 \mathrm{~cm} / \mathrm{s}$ were independent predictors. Other clinical and echocardiographic parameters had no relationship with the outcome.

Fig 1 shows the Kaplan-Meier curves in the patients when they were divided into 2 or 3 groups based on the various TEE-derived parameters. As shown, patients with severe spontaneous echo contrast or LAA velocity $<20 \mathrm{~cm} / \mathrm{s}$ or with LAA thrombus had a poorer prognosis compared with those without these abnormalities. No difference in prognosis was observed between patients with and without LAA enlargement.

\section{Discussion}

We prospectively examined the prognostic value of the TEE-derived parameters that imply a risk of thromboembolism in patients with chronic nonvalvular AF, and found that LAA velocity $<20 \mathrm{~cm} / \mathrm{s}$, LA spontaneous echo contrast and LAA thrombus, in particular, were significant predictors. Our results are consistent with those of previous studies ${ }^{7-10}$ and provide insights into the pathophysiology of thromboembolism in these patients and the potential clinical role of TEE-based risk stratification.

\section{Comparison With Previous Studies}

Few data are available on the TEE features that are linked to the risk of thromboembolism in patients with AF6-10 Previous studies have shown that echocardiographic parameters based on the transthoracic method, including LA size and left ventricular function, are useful for assessing embolic potential; 1,5 however, neither of these were independent predictors of embolic events in our study, which may be attributed to the relatively small number of patients and differences in the clinical and echocardiographic characteristics. The method we used for assessing LA size, which was based on the anteroposterior diameter only, might also explain this.

It is well known that LAA dysfunction and LA spontaneous echo contrast are associated with thromboembolism. $6,8,10$ Leung et $\mathrm{al}^{6}$ showed that spontaneous echo contrast was the only significant predictor of death or stroke in patients with nonvalvular AF and the annual event rate of thromboembolism in their patients with spontaneous echo contrast was $12 \%$, in contrast to $4.4 \%$ in ours. This discrepancy may be related to the fact that a much lower proportion of patients in that study (34\% of patients, even with spontaneous echo contrast) were receiving anticoagulation therapy. More recently, Kamp et al demonstrated that LAA velocity $<20 \mathrm{~cm} / \mathrm{s}$ could be used to identify patients at increased risk of subsequent thromboembolism8, and in cross-sectional studies, Chimowitz et al ${ }^{18}$ and others ${ }^{10,19}$ found that nonvalvular AF patients with spontaneous echo contrast and/or LAA dysfunction had increased risks of stroke or peripheral embolism compared with those without these features. In the SPAF-III trial, patients with an LAA velocity $<20 \mathrm{~cm} / \mathrm{s}$, those with dense spontaneous echo contrast, and those with LAA thrombus were at the highest risk of thromboembolism, ${ }^{7}, 17$ as was seen in our study.

Most of the previous reports failed to find LAA size an important predictor of embolic events in patients with nonvalvular $\mathrm{AF} 7,8$ and that was the case in the present study. Only one study has shown that the length and width of the LAA, not the area, are significant TEE parameters for predicting thromboembolism ${ }^{9}$ and those findings suggest that functional state, rather than morphological change, of the LAA contributes to thrombus formation and subsequent embolism.

\section{Pathophysiological Basis for Thromboembolism}

In the present study, 5 of 16 patients with LAA thrombus developed thromboembolism despite an adjusted dose of warfarin therapy, giving an event rate of $13 \%$ per year. Moreover, 6 of 7 patients with this complication had LAA thrombus. Although the high event rate in this study might be related to a strong selection bias in the patients referred for TEE (more patients with prior embolic event were included), this finding suggests that most of the ischemic events are caused by LAA blood stagnation, and that patients who have LAA thrombus may remain at high risk for thromboembolism during anticoagulation therapy.

It has been reported that TEE parameters remain strong predictors even when anticoagulation therapy is included as an independent predictor. The prospective SPAF-III study showed that despite an adjusted dose of warfarin therapy (INR 2.0-3.0), patients with LAA thrombus was at high risk (17.9\% annual embolic event rate)? Likewise, the Embolism in Left Atrial Thrombi Group observed that 4 of 10 patients who were receiving optimal anticoagulation therapy had a stroke? These data, as well as ours, raise the following questions: What mechanism underlies the embolic event in patients with LAA thrombus who are on anticoagulation therapy? What is the next strategy for treating such highrisk patients?

Some of the ischemic attacks may have resulted from systemic atherosclerosis because aortic plaque is the strongest predictor of future thromboembolism in nonvalvular AF? As shown in previous studies by us and by others, ${ }^{7-9,14,17}$ patients with LAA thrombus frequently have LAA dysfunction and severe spontaneous echo contrast, both of which are also thrombogenic signs. Therefore, multiple interrelated factors might outweigh the beneficial effect of warfarin, resulting in thromboembolism. A recent report has shown that fluctuation of the anticoagulation effect is related to the development of stroke in patients with nonvalvular AF, and therefore concluded that intensive therapy does not always reduce the risk of thromboembolism? 20 We also speculate that anticoagulation is not enough to resolve LAA thrombus within a stagnant LAA, compared with the peripheral vessels. In addition to Virchow's triad, localized blood stagnation is associated with localized activation of coagulability.21 Nevertheless, patients with $\mathrm{AF}$ and LAA thrombus may require more intensive anticoagulation, and a large-scale prospective study is needed to address this issue.

\section{Study Limitations}

The present study did not include one potential risk factor of thromboembolism, 'aortic plaque'7,17 as this parameter had not been standardized at the beginning of our study. The somewhat light level of anticoagulation therapy used in the present patients compared with previous studies ${ }^{7,9}$ (INR 1.8-2.5 vs 2.0-3.0) cannot rule out the high rate of embolic event in the patients with LAA thrombus. Also, variation in the anticoagulation intensity, which we did not assess, might have influenced the results. ${ }^{20}$ In the present study, the cutoff points for various echocardiographic parameters were made according to the SPAF-III trial $^{7}$ and others ${ }^{8,17}$ and other choices for cutoff points might have led to different results. Finally, our patients were selective in 
population because the decision to perform TEE depended on the primary physicians.

\section{Conclusions}

We determined whether TEE-derived parameters could predict thromboembolism in 89 patients with chronic nonvalvular AF. After a follow-up period of $29 \pm 10$ months, 7 patients had experienced thromboembolism. Multivariate analysis revealed that LAA dysfunction and LAA thrombus were significant predictors of the outcome. Therefore, TEE parameters, particularly the presence of LAA thrombus, can be used to assess thromboembolic potential in patients with chronic nonvalvular AF.

\section{References}

1. Risk factors for stroke and efficacy of antithrombotic therapy in atrial fibrillation: Analysis of pooled data from five randomized controlled trials. Arch Intern Med 1994; 154: 1449-1457

2. Blackshear JL, Kopecky SL, Litin SC, Safford RE, Hammil SC: Management of atrial fibrillation in adults: Prevention of thromboembolism and symptomatic treatment. Mayo Clin Proc 1996; 71: $150-160$

3. Moulton AW, Singer DE, Haas J S: Risk factors for stroke in patients with nonrheumatic atrial fibrillation: A case-control study. Am J Med 1991; 91: $156-161$

4. Flegel KM, Hanley J: Risk factors for stroke and other embolic events in patients with nonrheumatic atrial fibrillation. Stroke 1989; 20: $1000-1004$

5. The Stoke Prevention in Atrial Fibrillation Investigators: Predictors of thromboembolism in atrial fibrillation, II: Echocardiographic features of patients at risk. Ann Intern Med 1992; 116: 6-12

6. Leung DY, Black IW, Cranney GB, Hopkins AP, Walsh WF: Prognostic implications of left atrial spontaneous echo contrast in nonvalvular atrial fibrillation. J Am Coll Cardiol 1994; 24: 755-762

7. The Stroke Prevention in Atrial Fibrillation Investigators Committee on Echocardiography: Transesophageal echocardiographic correlates of thromboembolism in high-risk patients with nonvalvular atrial fibrillation. Ann Intern Med 1998; 128: 639-647

8. Kamp O, Verhorst PMJ, Welling RC, Visser CA: Importance of left atrial appendage flow as a predictor of thromboembolic events in patients with atrial fibrillation. Eur Heart J 1999; 20: 979-985

9. Stöllberger C, Chnupa P, Kronik G, Brainin M, Finsterer J, Schneider
B, et al: Transesophageal echocardiography to assess embolic risk in patients with atrial fibrillation. Ann Intern Med 1998; 128: 630-638

10. Igarashi Y, Kasai H, Yamashita F, Sato T, Inuzuka H, Ojima K, et al: Lipoprotein(a), left atrial appendage function and thromboembolic risk in patients with chronic nonvalvular atrial fibrillation. Jpn Circ J 2000; 64: $93-98$

11. Sahn JD, DeMaria A, Kisslo J, Weyman A: Recommendations regarding quantitation in M-mode echocardiography: Results of a survey of echocardiographic measurements. Circulation 1978; 58: 1072-1083

12. Beppu S, Nimura Y, Sakakibara H, Nagata S, Park YD, Izumi S: Smoke-like echo in the left atrial cavity in mitral valve disease: Its features and significance. J Am Coll Cardiol 1985; 6: 744-749

13. Klein AL, Murray RD, Black IW, Chandra S, Grimm RA, Leung DY, et al: Integrated backscatter for quantification of left atrial spontaneous echo contrast. J Am Coll Cardiol 1996; 28: 222-231

14. Ito T, Suwa M, Kobashi A, Yagi H, Nakamura T, Miyazaki S, et al: Integrated backscatter assessment of left atrial spontaneous echo contrast in chronic atrial fibrillation: Relation with clinical and echocardiographic parameters. J Am Soc Echocardiogr 2000; 13: 666-673

15. Pollick C, Taylor D: Assessment of left atrial appendage function by transesophageal echocardiography: Implications for the development of thrombus. Circulation 1991; 84: 223-231

16. Ito T, Suwa M, Hirota Y, Otake Y, Moriguchi A, Kawamura K: Influence of left atrial function on Doppler transmitral and pulmonary venous flow patterns in dilated and hypertrophic cardiomyopathy: Evaluation of left atrial appendage function by transesophageal echocardiography. Am Heart J 1996; 131: $122-130$

17. Zabalgoitia M, Halperin JL, Pearce LA, Blackshear JL, Asinger RW, Hart RG: Transesophageal echocardiographic correlates of clinical risk of thromboembolism in nonvalvular atrial fibrillation. $J$ Am Coll Cardiol 1998; 31: 1622-1626

18. Chimowitz MI, DeGeorgia MA, Poole RM, Hepner A, Armstrong WM: Left atrial spontaneous echo contrast in highly associated with previous stroke in patients with atrial fibrillation or mitral stenosis. Stroke 1993; 24: 1015-1059

19. Tsai LM, Chen JH, Fang CJ, Lin LJ, Kwan CM: Clinical implications of left atrial spontaneous echo contrast in nonrheumatic atrial fibrillation. Am J Cardiol 1992; 70: 327-331

20. Nozawa T, Asanoi H, Inoue H, TOWARD investigators: Instability of anticoagulation intensity contributes to occurrence of ischemic stroke in patients with non-rheumatic atrial fibrillation. Jpn Circ J 2001; 65: 404-408

21. Peverill RE, Harper RW, Gelman J, Gan TE, Harris G, Smolicj JJ: Determinants of increased regional left atrial coagulation activity in patients with mitral stenosis. Circulation 1996; 94: 331-339 